\title{
Additional possibilities of chimeric antigen receptor T-cells in B-cell lymphoma: combination therapy
}

\author{
Yan Yang ${ }^{1,2}$, Jing Zhou ${ }^{1}$, Cong Cao ${ }^{1}$, Panpan Cai ${ }^{1}$, Xinxuan Wang ${ }^{1}$, Chun Chang ${ }^{1}$, Jingxuan Wang ${ }^{1}$, \\ Qingyuan Zhang ${ }^{1}$
}

${ }^{1}$ Department of Medical Oncology, Harbin Medical University Cancer Hospital, Harbin, China; ${ }^{2}$ Depatment of Medical Oncology, Jilin Cancer Hospital, Changchun, China

Contributions: (I) Conception and design: Y Yang, J Wang; (II) Administrative support: Q Zhang; (III) Provision of study materials or patients: Y Yang, J Zhou; (IV) Collection and assembly of data: Y Yang, J Zhou; (V) Data analysis and interpretation: J Wang; (VI) Manuscript writing: All authors; (VII) Final approval of manuscript: All authors.

Correspondence to: Jingxuan Wang; Qingyuan Zhang. Department of Medical Oncology, Harbin Medical University Cancer Hospital, 150 Haping Road, Harbin 150040, China. Email: wjxilywendy@126.com; zqywsci@163.com.

\begin{abstract}
B-cell non-Hodgkin's lymphoma (B-NHL) is a lymphoproliferative disorder that affects B lymphocytes. Chimeric antigen receptor (CAR) T-cell immunotherapy is a new type of immunotherapy that uses genetic engineering techniques to modify and expand the patient's autoimmune cells in vitro, after which these cells are reinfused into the patient. CAR-T cell immunotherapy has the potential to treat different types of B-cell lymphoma. Many clinical studies have shown that CAR-T cell therapy has significant antitumor effects on B-cell lymphoma. Although much work has been carried out to improve the efficacy of CAR-T cell therapy and to reduce associated side effects, there are still many issues to address. CAR-T cell therapy shows significant promise in treating B-NHL, but some patients still have a poor initial response to this therapy where the infused CAR-T cells show insufficient persistence. With the rapid development of immunological therapy, combination therapy has been certified to improve the efficacy of CAR-T cell therapy. Targeted drugs such as programmed death-1 (PD-1) inhibitors, programmed cell death-ligand 1 (PD-L1) inhibitors, and Bruton's tyrosine kinase (BTK) inhibitors may further enhance the efficacy and reduce the side effects of CAR-T cell treatment. This article reviews the rationale and relevant clinical research on combination therapy based on CAR-T cell therapy for B-cell lymphoma treatment.
\end{abstract}

Keywords: CAR-T; immunotherapy; B-cell lymphoma; combination therapy

Submitted Jan 02, 2020. Accepted for publication Sep 07, 2020.

doi: $10.21037 /$ tcr-20-72

View this article at: http://dx.doi.org/10.21037/tcr-20-72

\section{Introduction}

Chimeric antigen receptor (CAR) T-cell immunotherapy is a new type of immunotherapy that uses genetic engineering techniques to modify and expand the patient's autoimmune cells in vitro, after which these cells are reinfused into the patient (1). Compared to traditional immunotherapy, CAR-T cell immunotherapy has many advantages, such as stronger targeting, a wider range of actions, longer-lasting effects, and stronger maneuverability $(2,3)$. It can recognize relevant antigens and act on tumor cells, killing and clearing them (4). CAR-T cell therapy has produced encouraging results in the treatment of hematological diseases (5). Many studies have confirmed that CAR-T cell therapy shows strong antitumor effects in different types of B-cell lymphomas.

B-cell non-Hodgkin's lymphoma (NHL) is a lymphoproliferative disorder that affects B lymphocytes. The most common subtypes of B-cell NHL (B-NHL) are diffuse large B-cell lymphoma (DLBCL), chronic lymphocytic leukemia (CLL)/small lymphocytic lymphoma, follicular lymphoma (FL), marginal zone lymphoma, and mantle cell lymphoma (MCL). The treatment provided to 
B-NHL patients is currently undergoing a revolutionary shift. Twenty years ago, rituximab revolutionized the treatment of $\mathrm{CD} 20^{+} \mathrm{B}$-cell hematological malignancies, and the need for conventional chemotherapy continues to decline as more immunotherapeutic approaches become available. The addition of rituximab to CHOP (cyclophosphamide, doxorubicin, vincristine, and prednisone) increased the percentage of cases with complete remission (CR) from $63 \%$ to $76 \%, 2$-year eventfree survival (EFS) from $38 \%$ to $57 \%$, and overall survival (OS) from $57 \%$ to $70 \%$ (6). At present, R-CHOP (CHOP plus rituximab) is still the standard first-line treatment for many types of B-NHL: $50 \%$ to $60 \%$ of patients treated with R-CHOP achieve clinical cure. However, $30 \%$ to $40 \%$ of patients will relapse. Patients with relapsed/refractory disease are treated with second-line salvage chemotherapy. Those who respond then receive consolidation highdose chemotherapy with autologous stem cell transplant (HDC/ASCT). Patients unsuitable for HDC/ASCT may receive subsequent lines of chemotherapy. Additionally, the introduction of targeted drugs, such as programmed death-1 (PD-1)/programmed cell death-ligand 1 (PD-L1) inhibitors, Bruton's tyrosine kinase (BTK) inhibitors, and histone deacetylases (HDACs) inhibitors, has shown exciting results in relapsed and refractory B-cell lymphoma patients (7).

Although CAR-T cell therapy shows significant promise in treating B-NHL, some patients still have a poor initial response to this therapy where the infused CAR-T cells show insufficient persistence. Multiple mechanisms may lead to CAR-T cell failure, including tumor microenvironment (TME) immunosuppression and tumorintrinsic properties associated with antigen or inhibitory ligand expression, as well as the attributes of adoptively transferred T-cells. When CAR-T cells are infused into patients, these cells often encounter inhibitory TMEs. CAR-T cells and inhibitory ligands can bind to inhibitory receptors on T-cells and hinder the antitumor response of these cells. Some studies suggest this is mediated by the patient's immune system responding to self-reengineered T-cells through checkpoint pathway activity, resulting in loss of function or physical loss of these cells. The efficacy of CAR-T cell therapy is related to the proliferative capacity of T-cells in vivo and the persistence of genetically modified cells. Therefore, defining T-cell phenotypes can influence antitumor activity. Pharmacological inhibition of protein kinase B (AKT), mammalian target of rapamycin (mTOR), or glycolysis during CAR-T cell expansion can promote effector memory T-cell formation. However, inhibition of these pathways also reduces the proliferative capacity of CAR-T cells, limiting therapeutic cell expansion. In summary, combination therapy may bring fresh hope for patients who show poor initial response to CAR-T cell therapy, as well as those with relapsed and refractory B-cell lymphoma.

Mohty et al. addressed the concept of CAR T cell therapy and indicated its role in the evolving landscape of management of patients with refractory/relapsed diffuse large B cell lymphoma (8). Hopfinger et al. summarized the results of three large phase II CD19 CAR-T cell trials and focus on AEs (9). In this review, we summarize the principles and relevant clinical research on therapy that combines CAR-T cell therapy and multiple targeted drugs for the treatment of B-cell lymphoma.

\section{Overview of CAR-T cell therapy}

\section{CAR-T cell structure}

CARs are recombinant receptors that target specific surface molecules. CARs comprise three structural components: an extracellular antigen-binding domain; a transmembrane domain; and a intracellular signal domain (10). Different designs of these three components may affect CAR-T cell function. The extracellular domain, a single-chain variable fragment $(\mathrm{scFv})$, is composed of both light and heavy chains, which are responsible for antigen recognition. This recognition involves the specific binding of antibodies to antigens. scFv designed to recognize antigens include CD19, CD20, epidermal growth factor receptor (EGFR), Her2/ neu, GD2, prostate-specific membrane antigen (PSMA), and receptor tyrosine kinase-like orphan receptor 1 (ROR1). The transmembrane domain connects the intracellular and extracellular domains. It is generally composed of dimeric membrane proteins (DMC) that anchor CARs to the T-cell membrane. Different designs of the transmembrane domain affect the expression of introduced CARs. The intracellular signaling domain employs immune-receptor tyrosine-based activation motifs (ITAMs), usually TCR $(\mathrm{CD}-3 \zeta)$ or Fc receptor $\gamma$-chain $(\mathrm{FcR} \gamma)$, which transduces T-cell receptor (TCR)-like signals into the cell when the extracellular domain $\mathrm{scFv}$ binds to the target antigen (11).

\section{CAR-T cell therapy treatment process}

CAR-T cell therapy begins with the collection of mononuclear cells from the patient's peripheral blood using a blood cell separator. Then, the patient's mononuclear cells 
are transferred to a cell processing center, where selective $\mathrm{T}$-cells are activated in a proliferative environment. CAR-T cells are generated by transfecting CAR into T-cells using retroviral or lentiviral vectors. Expanded CAR-T cells are then returned to the treatment institution and infused into the patient, in a process that usually takes two to three weeks (12). Before the CAR-T cell expansion process, physicians often adopt bridging chemotherapy to avoid rapid disease progression and maintain the patient's general condition. This can decrease the number of T-cells (including regulatory T-cells) in vivo, which upregulates cytokines such as IL-7 and IL-15. These cytokines promote T-cell expansion, including CAR-T cells, and enhance the antitumor activity of CAR-T cells (13).

\section{Progress in the development of CAR-T cell therapy}

Since the first reported case of anti-CD19 CAR-T cell therapy in 2010, it has been aggressively researched and was finally approved by the US Food and Drug Administration (FDA) in 2017 (14). First-generation CAR-T cell therapy involves only one intracellular signaling region and exhibits weak antitumor effects (15). Second-generation CAR-T cell therapy enhances its antitumor effects by adding costimulatory domains CD28 $(16)$ or $4-1 \mathrm{BB}(17,18)$ to first-generation CAR-T cell therapy. Third-generation CAR-T cell therapy comprises an activation domain and multiple costimulatory domains, such as CD27, CD28, and OX40 (19). The addition of these domains not only enhances the ability of CAR-T cells to recognize and bind to tumor-associated antigens (TAAs), but also significantly improves their ability to kill tumor cells. Fourth-generation CAR-T cell therapy introduces proinflammatory cytokines, such as IL-12, and costimulatory ligands (4-1BBL and CD40L) (20,21). This releases proinflammatory factors, and then recruits and activates more immune cells in the immunosuppressive microenvironment to produce more extensive antitumor immune effects (22-24). Of these four types of CAR-T cell therapy, second-generation CAR-T cell therapy is used most frequently because of its stability and controllable lateral function, and it is the most established of these therapies.

\section{Progression of CAR-T cell therapy in the treatment of B-cell lymphoma}

Despite improvements made in chemoimmunotherapy, NHL is the most common hematologic malignancy.
After first-line therapy, a significant number of patients experience disease progression or relapse. Anti-CD19 CAR-T cell therapy is considered as the most promising and effective therapy for overcoming refractory and relapsed B-NHL (25). Currently, there are two US FDA-approved anti-CD19 CAR-T cell therapies for the treatment of patients with relapsed and refractory aggressive NHL: tisagenlecleucel and axicabtagene ciloleucel (axi-cel). A third product, lisocabtagene-maraleucel (liso-cel), is undergoing clinical trials, and we await the preliminary results of these trials (26-32).

Tisagenlecleucel is a second-generation CAR-T cell therapy that uses CD8 as a transmembrane domain and $4-1 \mathrm{BB}$ as a costimulatory domain. This therapy was developed by the University of Pennsylvania (UPenn) in collaboration with Novartis and was approved by the FDA in 2017 for pediatric B-cell acute lymphoblastic leukemia (B-ALL) (26,31). UPenn conducted a pilot study of tisagenlecleucel in patients with B-cell lymphoma. Twentyeight patients were evaluable in this study (DLBCL, $n=14$; FL, $n=14$ ), and the primary study endpoint was overall response rate (ORR) at 3 months. At approximately 3 months post-infusion, the ORR was $50 \%(7 / 14)$ in patients with DLBCL and 79\% (11/14) in patients with FL, with $57 \%(16 / 28)$ achieving CR. The 16 patients (DLBCL, $\mathrm{n}=6 ; \mathrm{FL}, \mathrm{n}=10$ ) who achieved CR at 6 months post-infusion had a durable response, with a median followup of 29.3 months. Notably, 4 patients (DLBCL, n=1; FL, $\mathrm{n}=3$ ) who had achieved partial response (PR) at 3 months had achieved CR at 6 months post-infusion. Subsequently, a phase II clinical trial of tisagenlecleucel was conducted in patients with relapsed and refractory DLBCL (JULIET; NCT02345248) (26,33). CAR-T cells were infused into 111 patients, of whom 93 were evaluable. The best ORR was $52 \%$ (48/93) and the CR rate was $40 \%$ (37/93). The follow-up data was presented at the $60^{\text {th }}$ Annual Meeting of the American Society of Hematology (ASH) in 2018. The median duration of response and the OS of the CR patients was not reached within the median follow-up of 19.3 months (34). Recurrence-free survival was $64 \%$ at 12 and 18 months for all patients who responded to the therapy. Based on these promising results, the US FDA approved tisagenlecleucel for the treatment of relapsed and refractory DLBCL in May, 2018.

Axi-cel, which uses CD28 as the transmembrane domain and activation domain, was originally developed by the National Cancer Institute (NCI). In the ZUMA-1 trial (NCT02348216), a study of axi-cel in refractory B-NHL, 
of the 119 patients enrolled, 108 patients received axi-cel infusion $(35,36)$. There were 101 evaluable patients, who were followed up for a median of 27.1 months. Of the 101 patients, 84 (83\%) had an objective response to axi-cel, 59 (58\%) achieved CR, and 25 (25\%) attained PR. Ten patients $(10 \%)$ maintained stable disease (SD), five (5\%) progressed to best response, and two (2\%) were not assessable (35). A single infusion of axi-cel in many patients with relapsed and refractory B-NHL resulted in a durable response lasting more than two years without further consolidation therapy. The expected median OS with conventional chemotherapy is approximately six months and the two-year OS rate is approximately $20 \%$. The best ORR in this study was significantly higher than that of relapsed and refractory DLBCL treated with conventional chemotherapy, the historical control (37). Axi-cel was approved by the US FDA in October, 2017, for the treatment of relapsed and refractory DLBCL when relapse occurs after at least two lines of therapy, and was approved by the European Medicines Agency (EMA) in June, 2018.

Liso-cel uses CD28 as the transmembrane domain and $4-1 \mathrm{BB}$ as a costimulatory domain. Juno and Celgene conducted a US multicenter phase I study of liso-cel called Transcend NHL001, which initially enrolled patients with various subtypes of aggressive B-NHL, and subsequently expanded the cohort to enroll patients with DLBCL, double-triple hit lymphoma, and transformed follicular lymphoma. Updated results of Transcend NHL001 were presented at the American Society of Clinical Oncology (ASCO) Meeting in 2018. Thirty-seven patients with relapsed and refractory DLBCL received a critical dose of liso-cel, with an ORR of $49 \%$ and a CR rate of $46 \%$ at 6 months (38). Notably, toxicities were well managed, with only 1 patient developing grade 3 cytokine release syndrome (CRS) and 13 patients experiencing grade 3 or 4 neurotoxicity.

\section{Combination of CAR-T cell therapy and targeted agents in the treatment of lymphoma}

\section{CAR-T cell therapy in combination with PD-1 inbibitors}

PD-1 is an inhibitory receptor expressed by activated T-cells, activated B-cells, natural killer (NK) cells, and myeloid cells $(39,40)$. PD-L1 is widely expressed in many somatic cells exposed to pro-inflammatory cytokines, and intratumoral inflammation-induced PD-L1 expression leads to PD-1mediated T-cell exhaustion, which suppresses the antitumor immunity of cytotoxic T-cells (41-43). Engagement of the
PD-1/PD-L1 pathway leads to the phosphorylation of the tyrosine motif in the cytoplasmic tail of the PD-1 inhibitory receptor. Due to the resultant dephosphorylation of phosphatidylinositol 3-kinase (PI3K), this also promotes the recruitment of tyrosine protein phosphatase non-receptor type 11 (PTPN11). The inhibition of PI3K leads to downstream activation of the Rac-serine threonine protein kinase, which reduces T-cell activation, proliferation, and survival (44). CAR-T cell therapy is a promising approach for the treatment of refractory hematologic malignancies, as CAR-T cells can increase the expression of exhaustion markers, such as PD-1, Tim-3, and Lag-3 $(39,45)$. Blocking the activation of the PD-1/PD-L1 pathway has therefore been suggested as a therapeutic strategy for enhancing the antitumor efficacy of CAR-T cell therapy $(46,47)$.

One expected effect of CAR-T cell therapy is a significant decrease in B-cells post-therapy. The blockade or inhibition of these checkpoints by naturally occurring checkpoint mechanisms in the human immune system can prolong the action of CAR-T cells, and patients with persistent B-cell aplasia who are not treated with transplantation are at high risk of relapse (48). At the 2018 ASH Meeting, Dr. Maude presented the results of a small single-center study that included 14 children and adolescents with B-ALL who received pembrolizumab or nivolumab 2 to 7 weeks after an infusion of tisagenlecleucel. The B-cell counts of three patients recovered soon after the tisagenlecleucel infusion and fell again after the addition of PD-1 blockade, a marker of CAR-T cell function recovery. CR was later attained by all three of these patients. PD-1/PD-L1 inhibition helped seven patients in this study re-establish their initial response to tisagenlecleucel, and of four patients whose disease had spread beyond the bone marrow, two attained CR and two attained PR. Four patients who did not respond to CAR-T cell therapy did not attain CR when treated with pembrolizumab, but PR was observed. Dr. Maude and her team continue to follow these patients and explore strategies to improve health outcomes using CAR-T cell therapy in combination with immune checkpoint inhibitors (9).

In a small study conducted in China, researchers designed four experimental groups to investigate combination therapy for PD-1 overexpressing malignant lymphoma. The first group contained anti-CD19 CAR-T cells prepared from the peripheral blood of patients with PD-1 overexpressing malignant lymphoma. The second group also contained anti-CD19 CAR-T cells prepared from the peripheral blood of patients with $\mathrm{PD}-1$ overexpressing malignant lymphoma, but nivolumab was added at final 
concentrations of 72,36 , and $18 \mu \mathrm{g} / \mathrm{mL}$ on day 8 of cell expansion. The third group contained T-cells from patients with high peripheral blood PD-1 expression combined with $72 \mu \mathrm{g} / \mathrm{mL}$ of nivolumab; and the fourth group contained anti-CD19 CAR-T cells prepared from five healthy donors (HDs). The results of this study show that the transfection rate of anti-CD19 CAR-T cells in patients with high PD-1 expression was close to that of HDs $(32.80 \% \pm 7.22 \% v s$. $35.10 \% \pm 5.84 \%, \mathrm{P}=0.593)$. The combination of $72 \mu \mathrm{g} / \mathrm{mL}$ of nivolumab and anti-CD19 CAR-T cells showed better killing rates than either the combination of $72 \mu \mathrm{g} / \mathrm{mL}$ of nivolumab with patient-derived T-cells or the anti-CD19 CAR-T cells alone $(\mathrm{P}<0.001$ for both). The killing rate of the combination of $72 \mu \mathrm{g} / \mathrm{mL}$ of nivolumab and anti-CD19 CAR-T cells at 48 hours was $71.61 \% \pm 9.50 \%$; the killing rate of the combination of $72 \mu \mathrm{g} / \mathrm{mL}$ of nivolumab with patient-derived T-cells was $6.77 \% \pm 1.26 \%$; and the killing rate of anti-CD19 CAR-T cells alone was $15.33 \% \pm 4.11 \%$. Different doses of nivolumab in combination with patientderived anti-CD19 CAR-T cells did not affect the levels of inflammatory cytokines IFN- $\gamma$ and TNF- $\alpha(\mathrm{P}>0.05$ for all). This study showed that nivolumab at a final concentration of $36 \mu \mathrm{g} / \mathrm{mL}$ combined with anti-CD19 CAR-T cells reduced side effects of the drug while enhancing the killing activity of anti-CD19 CAR-T cells (49); however, studies with larger sample sizes are needed to confirm these results.

Schuster, Riedell, and Joseph McGuirk from the University of Kansas Cancer Center are in the process of testing the safety and effectiveness of pembrolizumab administered to patients with DLBCL several weeks after treatment with tisagenlecleucel, rather than in response to patients relapsing or failing to respond to CAR-T cell therapy (9). We await the results of this ongoing study.

\section{CAR-T cell therapy in combination with BTK inbibitors}

BTK is a Tec family kinase present in the cell membrane and nucleus, and it is an essential component of the B-cell receptor (BCR) signaling pathway. BTK plays an important role in $\mathrm{B}$-cell maturation and regulation of cellular processes, such as cell differentiation, cell division, and signal transduction, and this kinase is highly expressed in B-cell malignancies (50). Ibrutinib is a covalent irreversible inhibitor of BTK that is highly selective for this kinase and has been approved by the US FDA and the EMA for the treatment of CLL (51). BTK inhibitors can theoretically increase the efficiency of CAR-T cell therapy, enhancing its overall antitumor effect by reducing cell terminal differentiation through the inhibition of AKT signaling, which in turn increases the proportion of memory CAR-T cells (52). In addition, ibrutinib effectively mobilizes tumorinfiltrating B-cells to infiltrate peripheral blood so they can be destroyed by circulating CAR-T cells.

A recent study combined ibrutinib with anti-CD19 CAR-T cell therapy (tisagenlecleucel), anticipating an improved response in MCL (53). Combining MCL cell lines, primary MCL samples, autologous or normal donor-derived anti-CD19 CAR-T cells, and ibrutinib, these researchers tested the effect of this combination in vitro and in a mouse xenograft model. The in vitro investigation showed that MCL cells strongly activate multiple effector functions of tisagenlecleucel, and that tisagenlecleucel kills MCL cells more effectively in the presence of ibrutinib. In the xenograft MCL model, mice treated with tisagenlecleucel showed a statistically significant improvement in lymphoma control compared to mice treated with ibrutinib. All mice treated with ibrutinib monotherapy died before day 100 , whereas tisagenlecleucel fostered long-term survival in recipient mice, which suggests that tisagenlecleucel is therapeutically more effective than ibrutinib in this model. When ibrutinib and tisagenlecleucel were administered in combination, $80 \%$ of the mice maintained long-term remission $(\mathrm{P}<0.05)$. This study demonstrated that when used in combination, these two therapies improve antitumor activity and exhibit a lower recurrence rate compared to monotherapy.

Gill et al. presented the results of a trial that introduced anti-CD19 CAR-T cell therapy into conventional CLL therapy for patients who did not achieve CR after six months of ibrutinib treatment at the 2017 ASCO Meeting (54). All patients received bridging chemotherapy, comprising bendamustine or fludarabine and cyclophosphamide, approximately one week before treatment. Subsequently, the researchers administered CAR-T cells and ibrutinib therapy to the patients. They later examined the patients' bone marrow CLL burden by flow cytometry and IGH gene rearrangements and used computed tomography imaging to evaluate the lymph nodes and spleen. After a median sixmonth follow-up, eight of nine evaluable patients achieved minimal residual disease (MRD) negativity as demonstrated by flow cytometry, and all patients had maintained bone marrow CR at their last follow-up. The nine evaluable patients displayed CRS at different levels: two patients with grade 1; six patients with grade 2; and one patient with grade 3. Overall, patients tolerated this regimen well and did not require corresponding treatment for side effects. 
As demonstrated by computer tomography, five patients attained CR, two patients attained PR, and two patients attained SD. However, the researchers indicated that longterm follow-up is needed to confirm the robustness of these results, and that further trials are required to investigate whether this combination therapy, when used as a firstline regimen for CLL, can spare patients from long-term treatment.

\section{CAR-T cell therapy in combination with PI3K inbibitors}

While the results of these CAR-T cell therapy studies are encouraging, there remain some lymphoma patients who do not respond. It has been shown that this phenomenon is associated with the infusion of low numbers of early memory T-cells (55). A phase II clinical trial of secondgeneration anti-CD19 CAR-T cell therapy containing CD28 costimulatory domains found that the frequency of CD62L-expressing central memory $\mathrm{T}\left(\mathrm{T}_{\mathrm{CM}}\right)$ cells in the infused product were significantly correlated with the volume of CARs in the peripheral blood, and that the frequency of these $T_{C M}$ cells was directly related to improved ORR and antitumor effects of the therapy (56). Therefore, a major goal for improving CAR-T cell therapy is generating $\mathrm{T}_{\mathrm{CM}}$-phenotype cells for patient infusion (57). T-cell expansion and differentiation is depended on the integration of TCRs, costimulatory molecules, and cytokine receptors (58). These signals converge to activate two major signal transduction networks within T-cells: the mitogenactivated protein kinase (MAPK) and phosphatidylinositol 3-kinase (PI3K)-AKT-mTOR pathways. These pathways are critical for T-cell activation, survival, expansion, and cytokine production, as well as $\mathrm{T}_{C M}$ cell formation and differentiation (59). The genetic and pharmacological disruption of extracellular signal-regulated kinases (ERKs), a distal component of the MAPK pathway, significantly impairs T-cell proliferation in both mice (60) and humans (61). In contrast, the proliferation and survival of murine CD8 T-cells is not impaired by the deletion of PI3CD, the gene that encodes the p110 catalytic subunit of PI3K, or the inhibition of AKT (62). Therefore, pharmacological interference of AKT signaling results in T-cell activation and expansion in human peripheral blood, as well as efficient retroviral transduction by means of CAR or TCR and promotion of CD62L expression (63). Additionally, glycolysis can enhance T-cell differentiation and lead to impaired antitumor effects in vivo. Therefore, the inhibition of AKT in CD19 CAR-T cell therapy can inhibit glycolysis and increase T-cell activity by interrupting the expression of key metabolic enzymes (55).

Idelalisib is a PI3K inhibitor that has been approved for the treatment of CLL and FL. As well as being able to eliminate malignant B-cells, idelalisib can exhaust regulatory T-cells, therefore reversing immune tolerance (64). In a previous study of CLL treatment, researchers combined idelalisib with anti-CD19 CAR-T cells. Peripheral blood samples were collected from $10 \mathrm{HDs}$ and 9 CLL patients (65). Their results demonstrated that, when cultivated with idelalisib, CD19-CAR expression increased significantly in both HDs $(59 \% \pm 12 \%$ vs. $64 \% \pm 13 \%, \mathrm{P}=0.002)$ and CLL patients $(65 \% \pm 13 \%$ vs. $73 \% \pm 11 \%, \mathrm{P}=0.02)$. On day 10 , malignant B-cells were markedly decreased in CLL patient samples and reached similar levels to HD-derived CAR-T cells. Idelalisib had no significant effect on the B-cells of HDs. Cytotoxic T-cells (CD3+/CD8+) and T helper cells (CD3+/ CD4+) derived from HDs and CLL patients expanded in different ways during cell cultivation. In HDs, T helper cell numbers decreased and cytotoxic T-cell numbers increased after introducing idelalisib. In contrast, CAR-T cells derived from CLL patients where idelalisib was not introduced contained more $\mathrm{T}$ helper cells and fewer cytotoxic T-cells. CD45RA + CCR7 + naive-like T (TN) cells positive for the memory-associated marker CD95 can be recognized by T memory stem cells (TSCM) in CAR-T cell products. The fraction of TN cells within CAR-T cells cultivated with idelalisib expressed higher levels of CD62L in both HDs and CLL patients; more than $95 \%$ of HD-derived cells expressed CD62L. Researchers used a xenograft NSG mouse model with human ALL cells (Nalm-6) to analyze the antitumor activity of anti-CD19 CAR-T cells in the presence or absence of idelalisib. This study ultimately showed that the antitumor activity of CAR-T cells was enhanced after idelalisib was introduced.

Decreased T-cell counts and an abnormal T-cell phenotype involving significantly increased proportions of CD27 CD28- T-cells, which is a hallmark of senescence, have been observed in heavily pretreated DLBCL patients. This may lead to insufficient yield or poor CAR-T cell quality during cell production (66). In another study, low concentrations of idelalisib and VIPhyb, a VIP pathway antagonist, were introduced into a mouse model. This significantly improved viable T-cell counts and delayed terminal differentiation (67). Conversely, the cytotoxicity of CAR-T cells against lymphomas was enhanced, which suggests that PI3K and VIP signal interruption is a promising method for limiting T-cell depletion during 
the expansion of CAR-T cells and improving the clinical efficacy of genetically modified cells.

\section{CAR-T cell therapy in combination with HDAC inhibitors}

HDACs are enzymes that deacetylate the acetyl group from $\varepsilon-\mathrm{N}$-acetyl lysine amino acids to produce histone, which leads to the tight wrapping of DNA. This enzyme can promote chromatin compaction and repress transcription of their related genes (68). HDAC inhibitors (HDACis) have been shown to enhance the levels of CD20-expressing protein and mRNA in Burkitt's lymphoma, which in turn enhances the expression of CD20 antigens on the surface of malignant B-cells (69). Pretreatment with HDACis before CD20 CAR-T cell therapy can enhance cytotoxic activity, and increased toxicity toward tumor cells and prolonged overall survival in a mouse cell model of Burkitt's lymphoma (70).

Another study showed that romidepsin combined with anti-CD20 CAR modified expanded peripheral blood NK (anti-CD20 CAR exPBNK) cells significantly induced cell death in rituximab sensitive and resistant Burkitt's lymphoma cells in vitro, and reduced tumor burden in mice xenografted with human Burkitt's lymphoma, improving their progress (71). This study demonstrated that romidepsin could enhance the antitumor activity of NK cells and anti-CD20 CAR exPBNK in rituximab sensitive and resistant Burkitt's lymphoma.

\section{CAR-T cell therapy in combination with rituximab}

Rituximab has greatly improved the outcomes of most B-NHL patients $(72,73)$. However, it is reported that B-NHL cells may lose CD20 expression after rituximab treatment, and this potential absence may result in resistance to rituximab $(74,75)$. Conversely, anti-CD20 CAR-T cells can provide alternative targets that can be treated sequentially with rituximab, or else rituximab can be used with anti-CD19 CAR-T cells to target multiple antigens simultaneously, therefore reducing the risk of immune escape (76). B-cell exhaustion after rituximab treatment is beneficial, in that it can maintain anti-CD20 CAR-T cell survival (77) and allow T-cells to efficiently transfer to B-NHL patients and remain at the lesion location. In another study, researchers attempted to investigate the cytotoxic effects of rituximab in combination with either anti-CD19 CAR-T or anti-CD38 CAR-T in xenografted B-NHL mice, and ultimately found that synergistic tumor inhibition continued for more than two months in these mice (78). We anticipate further encouraging results as investigations into combined rituximab and CAR-T cell therapy for different types of lymphoma continue.

A theoretical limitation of rituximab use in combination with CAR-T cell therapy is that residual serum rituximab may hinder CAR binding to CD20, which would impede T-cell mediated antitumor responses (76). Researchers identified a range of rituximab serum levels in CAR-T cell assays and ultimately found that, in the majority of patients, rituximab concentration was $100 \mathrm{mg} / \mathrm{mL}$ or less with a median concentration of more than $40 \mathrm{mg} / \mathrm{mL}$. In this concentration range, anti-CD20 CAR-T cells retain effective activity both in vitro and in vivo. Rituximab concentrations are significantly higher in CAR-T cell assays in mouse models than in most human patients. The results of mouse studies have indicated better health outcomes in mice that receive combination therapy compared to mice treated with CAR-T cells alone. Moreover, mice that receive combination therapy are not impaired by the rituximab. These experiments also clarify a point of clinical relevance: CAR-T cells may be effective in the treatment of rituximab-resistant tumors.

\section{CAR-T cell therapy in combination with lenalidomide}

Multiple myeloma (MM) is a hematological malignancy characterized by the clonal expansion of terminally differentiated B-cells (plasma cells) in the bone marrow. Its clinical characteristics include osteolytic bone disease, infection, and abnormal renal function. There are several ongoing clinical studies on CAR-T cell therapy in MM (79). In one such study, an MM patient was given a tisagenlecleucel infusion after a standard autologous transplantation. An important finding of this study was that tisagenlecleucel cells were still detectable in the blood and bone marrow up to several days postinfusion (80). The patient commenced lenalidomide maintenance therapy three months after infusion, and CR was still observed at the one-year follow-up. No signs of fever or other CRS were reported after tisagenlecleucel infusion. This study is ongoing, and we await the results of future follow-ups.

\section{Summary}

In recent years, CAR-T cell therapy for the treatment of both hematologic malignancies and solid tumors has 
advanced (81). However, it still faces many challenges: both T-cell exhaustion and immunological barriers limit the effectiveness of CAR-T cell therapy; and adverse events, including CRS and neurotoxicity, negatively impact treatment safety (82). Combination therapy based on CAR-T cell therapy may be a viable solution for these problems. Many targeted drugs have demonstrated excellent efficacy in the treatment of a variety of B-cell lymphomas. Combining CAR-T cell therapy with targeted drugs is therefore a promising strategy for the treatment of B-cell lymphoma. We summarized some trials in vivo and in vitro mentioned in this article of CAR-T cell therapy combination with targeted agents for the treatment of B-cell lymphoma in the Table 1 .

Table 1 In vivo and in vitro trials of CAR-T cell therapy in combination with targeted agents for the treatment of B-cell lymphoma

\begin{tabular}{|c|c|c|c|c|c|}
\hline $\begin{array}{l}\text { Combination } \\
\text { target }\end{array}$ & Drug & $\begin{array}{l}\text { CAR-T name/ } \\
\text { design/target }\end{array}$ & Disease & Intervention & Result \\
\hline PD-1 & $\begin{array}{l}\text { Pembrolizumab } \\
\text { or nivolumab }\end{array}$ & $\begin{array}{l}\text { Tisagenlecleucel/ } \\
\text { 4-1BB/CD19 }\end{array}$ & B-ALL & $\begin{array}{l}14 \text { children and } \\
\text { adolescents with B-ALL } \\
\text { received pembrolizumab } \\
\text { or nivolumab within } 2 \text { to } 7 \\
\text { weeks after the infusion of } \\
\text { tisagenlecleucel }\end{array}$ & $\begin{array}{l}3 \text { patients CR } \\
7 \text { patients re-established } \\
\text { their initial response to } \\
\text { tisagenlecleucel }\end{array}$ \\
\hline \multirow[t]{2}{*}{ PD-1 } & Nivolumab & $\begin{array}{l}\text { Anti-CD19 CAR-T } \\
\text { cell }\end{array}$ & In vitro & $\begin{array}{l}\text { Arm 1: anti-CD19 CAR-T } \\
\text { cells are prepared from } \\
\text { peripheral blood PD-1 } \\
\text { overexpressing malignant } \\
\text { lymphoma }\end{array}$ & $\begin{array}{l}\text { 1. The transfection rate of } \\
\text { anti-CD19 CAR-T cells in } \\
\text { patients with high PD-1 } \\
\text { expression was close to that } \\
\text { in the HDs }(32.80 \% \pm 7.22 \% \\
\text { vs. } 35.10 \% \pm 5.84 \%, P=0.593)\end{array}$ \\
\hline & & & & $\begin{array}{l}\text { Arm 3: T-cells from patients } \\
\text { with high peripheral blood } \\
\text { PD-1 expression combined } \\
\text { with } 72 \mu \mathrm{g} / \mathrm{mL} \text { nivolumab }\end{array}$ & $\begin{array}{l}\text { 3. The combination of } \\
\text { nivolumab at a final } \\
\text { concentration of } 36 \mu \mathrm{g} / \mathrm{mL} \\
\text { with anti-CD19 CAR-T cells } \\
\text { reduced the side effects of } \\
\text { the drug while enhancing the } \\
\text { killing activity of anti-CD19 } \\
\text { CAR-T cells }\end{array}$ \\
\hline BTK & Ibrutinib & $\begin{array}{l}\text { Anti-CD19 CAR-T } \\
\text { cell }\end{array}$ & In vitro & & $\begin{array}{l}\text { MCL cells strongly activated } \\
\text { the function of multiple } \\
\text { tisagenlecleucel effectors, } \\
\text { and the killing of MCL by } \\
\text { tisagenlecleucel was further } \\
\text { enhanced by ibrutinib }\end{array}$ \\
\hline
\end{tabular}

Table 1 (continued) 
Table 1 (continued)

\begin{tabular}{|c|c|c|c|c|c|}
\hline $\begin{array}{l}\text { Combination } \\
\text { target }\end{array}$ & Drug & $\begin{array}{l}\text { CAR-T name/ } \\
\text { design/target }\end{array}$ & Disease & Intervention & Result \\
\hline BTK & Ibrutinib & $\begin{array}{l}\text { Anti-CD19 CAR-T } \\
\text { cell }\end{array}$ & $\begin{array}{l}\text { Mouse } \\
\text { xenograft model }\end{array}$ & & $\begin{array}{l}\text { All mice treated with } \\
\text { ibrutinib monotherapy died } \\
\text { before day } 100, \text { whereas } \\
\text { tisagenlecleucel fostered } \\
\text { long-term survival of the } \\
\text { recipient mice. With the } \\
\text { combination of ibrutinib and } \\
\text { tisagenlecleucel, } 80 \% \text { of } \\
\text { mice maintained long-term } \\
\text { remission }(P<0.05)\end{array}$ \\
\hline \multirow[t]{2}{*}{ BTK } & \multirow[t]{2}{*}{ Ibrutinib } & \multirow[t]{2}{*}{$\begin{array}{l}\text { Anti-CD19 CAR-T } \\
\text { cell }\end{array}$} & \multirow[t]{2}{*}{$\begin{array}{l}9 \text { CLL patients } \\
\text { who did not } \\
\text { achieve CR } \\
\text { after } 6 \text { months } \\
\text { of Ibrutinib } \\
\text { treatment }\end{array}$} & & $\begin{array}{l}\text { Minimal residual disease } \\
\text { (MRD) negativity was } \\
\text { achieved on flow cytometry, } \\
\text { and all patients retained bone } \\
\text { marrow CR at last follow-up } \\
\text { CRS: grade } 1 \text { for } 2 \text { patients; } \\
\text { grade } 2 \text { for } 6 \text { patients; and } \\
\text { grade } 3 \text { for } 1 \text { patient }\end{array}$ \\
\hline & & & & & $\begin{array}{l}\text { CR in } 5 \text { patients, } P R \text { in } 2 \\
\text { patients, and SD in } 2 \text { patients }\end{array}$ \\
\hline PI3K & Idelalisib & Anti CD19 CAR-T & In vitro & $\begin{array}{l}\text { Arm 1: peripheral blood } \\
\text { samples were collected } \\
\text { from } 10 \text { HDs } \\
\text { Arm 2: peripheral blood } \\
\text { samples were collected } \\
\text { from } 9 \text { CLL patients }\end{array}$ & $\begin{array}{l}\text { The results showed that } \\
\text { cultivation with idelalisib } \\
\text { significantly increased CD19- } \\
\text { CAR expression in both } \\
\text { the HDs }(59 \% \pm 12 \% \text { vs. } \\
64 \% \pm 13 \%, P=0.002) \text { and the } \\
\text { CLL patients }(65 \% \pm 13 \% \text { vs. } \\
73 \% \pm 11 \%, P=0.02)\end{array}$ \\
\hline HDAC & Romidepsin & Anti-CD20 CAR-T & $\begin{array}{l}\text { A mouse } \\
\text { cell model } \\
\text { of Burkitt's } \\
\text { lymphoma }\end{array}$ & $\begin{array}{l}\text { Pretreatment with HDACis } \\
\text { before CD20 CAR-T } \\
\text { treatment }\end{array}$ & $\begin{array}{l}\text { The cytotoxic activity was } \\
\text { enhanced, the toxicity to } \\
\text { tumor cells was increased, } \\
\text { and overall survival was } \\
\text { prolonged }\end{array}$ \\
\hline HDAC & Romidepsin & $\begin{array}{l}\text { Anti-CD20 CAR } \\
\text { exPBNK }\end{array}$ & $\begin{array}{l}\text { A mouse } \\
\text { xenograft } \\
\text { humanized } \\
\text { Burkitt's } \\
\text { lymphoma } \\
\text { model }\end{array}$ & & $\begin{array}{l}\text { The antitumor activity of } \\
\text { natural NK cells and anti- } \\
\text { CD20 CAR exPBNK in } \\
\text { rituximab-sensitive and } \\
\text { resistant Burkitt's lymphoma } \\
\text { was enhanced }\end{array}$ \\
\hline CD20 & Rituximab & $\begin{array}{l}\text { Anti-CD19 CAR-T } \\
\text { or anti-CD38 CAR-T }\end{array}$ & $\begin{array}{l}\text { A xenograft } \\
\text { B-NHL mouse } \\
\text { model }\end{array}$ & & $\begin{array}{l}\text { Synergistic tumor inhibition } \\
\text { continued in mice for more } \\
\text { than } 2 \text { months }\end{array}$ \\
\hline
\end{tabular}


CAR-T cells frequently encounter inhibitory TMEs when infused into patients, in which cells and inhibitory ligands can bind to inhibitory receptors on T-cells and hinder their antitumor response. Checkpoint blockade therapies against PD-1, PD-L1, and CTL-4 use antibodies to disrupt interactions with inhibitory receptors on T-cells. This strategy of administering checkpoint blockade therapy after CAR-T cell treatment enhances the efficacy of CAR-T cell therapy in tumors with immunosuppressive TMEs.

The attributes of adoptively transferred T-cells themselves are also an important factor that affects the therapeutic effect of CAR-T cells. Ibrutinib primarily interferes with BCR signaling pathways by inhibiting BTK; however, it can affect multiple parts of the hematopoietic system, including T-cells. The theoretical basis for the binding of ibrutinib to CAR-T cells is its direct effect on T-cells and its ability to destroy immunosuppressive TMEs. PI3K delta has a role in both B-cell and T-cell function, and inhibition of this signaling pathway has direct antitumor activity in NHL and CLL. PI3K signaling is also important for the proliferation and function of T-cells.

At present, preclinical and limited clinical data shows that some binding of small molecules and monoclonal antibodies to CAR-T cells can overcome many drug resistance mechanisms and further improve the effectiveness of CAR-T cells. Although many combination approaches are mentioned in this paper, an exhaustive list is not included and many relevant clinical trials are ongoing; we eagerly anticipate future results. This new combination immunotherapy regimen merits further investigation to enhance its antitumor efficacy and minimize side effects.

\section{Acknowledgments}

Funding: This work is contributed by the colleagues of Harbin Medical University Cancer Hospital. We thank Dr. Zhang and Dr. Wang for the enlightening discussion. The research was supplied by the National Natural Science Foundation of China (Grant No. 81772813).

\section{Footnote}

Conflicts of Interest: All authors have completed the ICMJE uniform disclosure form (available at http://dx.doi. org/10.21037/tcr-20-72). The authors have no conflicts of interest to declare.

Ethical Statement: The authors are accountable for all aspects of the work in ensuring that questions related to the accuracy or integrity of any part of the work are appropriately investigated and resolved.

Open Access Statement: This is an Open Access article distributed in accordance with the Creative Commons Attribution-NonCommercial-NoDerivs 4.0 International License (CC BY-NC-ND 4.0), which permits the noncommercial replication and distribution of the article with the strict proviso that no changes or edits are made and the original work is properly cited (including links to both the formal publication through the relevant DOI and the license). See: https://creativecommons.org/licenses/by-nc-nd/4.0/.

\section{References}

1. Makita S, Imaizumi K, Kurosawa S, et al. Chimeric antigen receptor T-cell therapy for B-cell non-Hodgkin lymphoma: opportunities and challenges. Drugs Context 2019;8:212567.

2. Jurczak W, Długosz-Danecka M, Rivas Navarro F. The rationale for combination therapy in patients with aggressive B-cell non-Hodgkin lymphoma: ten questions. Future Oncol 2019;15:305-17.

3. Madden DL. From a Patient Advocate's Perspective: Does Cancer Immunotherapy Represent a Paradigm Shift? Curr Oncol Rep 2018;20:8.

4. SShah NN, Maatman T, Hari PN, et al. Multi targeted CAR-T cell therapies for B-cell malignancies. Front Oncol 2019;9:146.

5. Shah NN, Fry TJ. Mechanisms of resistance to CAR T cell therapy. Nat Rev Clin Oncol 2019;16:372-85.

6. Gerds AT, Michaelis LC, Shafer D. The Efficacy and Safety of the Addition of Rituximab to CHOP or a CHOP-Like Regimen In First Line Therapy for Diffuse Large B-Cell Lymphoma (DLBCL): A Meta-Analysis. Blood 2010;116:4913.

7. Heyman B, Yang Y. New developments in immunotherapy for lymphoma. Cancer Biol Med 2018;15:189-209.

8. Mohty M, Dulery R, Gauthier J, et al. CAR T-cell therapy for the management of refractory/relapsed high-grade B-cell lymphoma: a practical overview. Bone Marrow Transplant 2020;55:1525-32.

9. Hopfinger G, Jger U, Worel N. CAR-T Cell Therapy in Diffuse Large B Cell Lymphoma: Hype and Hope. Hemasphere 2019;3:e185.

10. Panagopoulou TI, Rafiq QA. CAR-T immunotherapies: Biotechnological strategies to improve safety, efficacy and 
clinical outcome through CAR engineering. Biotechnol Adv 2019;37:107411.

11. Eshhar Z, Waks T, Gross G, et al. Specific activation and targeting of cytotoxic lymphocytes through chimeric single chains consisting of antibody-binding domains and the gamma or zeta subunits of the immunoglobulin and T-cell receptors. Proc Natl Acad Sci U S A 1993;90:720-4.

12. Klebanoff CA, Khong HT, Antony PA, et al. Sinks, suppressors and antigen presenters: how lymphodepletion enhances T cell-mediated tumor immunotherapy. Trends Immunol 2005;26:111-7.

13. Highfill SL, Stroncek DF. Overcoming Challenges in Process Development of Cellular Therapies. Curr Hematol Malig Rep 2019;14:269-77.

14. Jensen MC, Popplewell L, Cooper LJ, et al. Antitransgene rejection responses contribute to attenuated persistence of adoptively transferred CD20/CD19-specific chimeric antigen receptor redirected $\mathrm{T}$ cells in humans. Biol Blood Marrow Transplant 2010;16:1245-56.

15. Kalos M, Levine BL, Porter DL, et al. T cells with chimeric antigen receptors have potent antitumor effects and can establish memory in patients with advanced leukemia. Sci Transl Med 2011;3:95ra73-95ra73.

16. Savoldo B, Ramos CA, Liu E, et al. CD28 costimulation improves expansion and persistence of chimeric antigen receptor-modified $\mathrm{T}$ cells in lymphoma patients. J Clin Invest 2011;121:1822-6.

17. Milone MC, Fish JD, Carpenito C, et al. Chimeric receptors containing CD137 signal transduction domains mediate enhanced survival of T cells and increased antileukemic efficacy in vivo. Mol Ther 2009;17:1453-64.

18. Long AH, Haso WM, Shern JF, et al. 4-1BB costimulation ameliorates $T$ cell exhaustion induced by tonic signaling of chimeric antigen receptors. Nat Med 2015;21:581-90.

19. Tang XY, Sun Y, Zhang A, et al. Third-generation CD28/4-1BB chimeric antigen receptor $T$ cells for chemotherapy relapsed or refractory acute lymphoblastic leukaemia: a non-randomised, open-label phase I trial protocol. BMJ Open 2016;6:e013904.

20. Pulè MA, Straathof KC, Dotti G, et al. A chimeric T cell antigen receptor that augments cytokine release and supports clonal expansion of primary human T cells. Mol Ther 2005;12:933-41.

21. Quintarelli C, Orlando D, Boffa I, et al. Choice of costimulatory domains and of cytokines determines CAR T-cell activity in neuroblastoma. Oncoimmunology 2018;7:e1433518.

22. Van Schandevyl S, Kerre T. Chimeric antigen receptor
T-cell therapy: design improvements and therapeutic strategies in cancer treatment. Acta Clin Belg 2020;75:26-32.

23. Ramos CA, Asgari Z, Liu E, et al. An inducible caspase 9 suicide gene to improve the safety of mesenchymal stromal cell therapies. Stem Cells 2010;28:1107-15.

24. Pegram HJ, Lee JC, Hayman EG, et al. Tumor-targeted T cells modified to secrete IL-12 eradicate systemic tumors without need for prior conditioning. Blood 2012;119:4133-41.

25. Kochenderfer JN, Wilson WH, Janik JE, et al. Eradication of B-lineage cells and regression of lymphoma in a patient treated with autologous $\mathrm{T}$ cells genetically engineered to recognize CD19. Blood 2010;116:4099-102.

26. Schuster SJ, Bishop MR, Tam CS, et al. Tisagenlecleucel in adult relapsed or refractory diffuse large B-cell lymphoma. N Engl J Med 2019;380:45-56.

27. Schuster SJ, Svoboda J, Chong EA, et al. Chimeric antigen receptor $\mathrm{T}$ cells in refractory B-cell lymphomas. N Engl J Med 2017;377:2545-54.

28. Porter DL, Levine BL, Kalos M, et al. Chimeric antigen receptor-modified $\mathrm{T}$ cells in chronic lymphoid leukemia. N Engl J Med 2011;365:725-33.

29. Kochenderfer JN, Dudley ME, Feldman SA, et al. B-cell depletion and remissions of malignancy along with cytokine-associated toxicity in a clinical trial of anti-CD19 chimeric-antigen-receptor-transduced T cells. Blood 2012;119:2709-20.

30. Grupp SA, Kalos M, Barrett D, et al. Chimeric antigen receptor-modified T cells for acute lymphoid leukemia. $\mathrm{N}$ Engl J Med 2013;368:1509-18.

31. Maude SL, Laetsch TW, Buechner J, et al. Tisagenlecleucel in children and young adults with B-cell lymphoblastic leukemia. N Engl J Med 2018;378:439-48.

32. Neelapu SS, Locke FL, Bartlett NL, et al. Axicabtagene Ciloleucel CAR T-Cell Therapy in Refractory Large B-Cell Lymphoma. N Engl J Med 2017;377:2531-44.

33. Borchmann $P$, Tam $C$, Jäger $U$, et al. editors. An updated analysis of JULIET, a global pivotal Phase 2 trial of tisagenlecleucel in adult patients with relapsed or refractory (r/r) diffuse large b-cell lymphoma (DLBCL). Oncology research and treatment; 2018: Karger Allschwilerstrasse 10, ch-4009 Basel, Switzerland.

34. Schuster SJ, Bishop MR, Tam C, et al. Sustained disease control for adult patients with relapsed or refractory diffuse large B-cell lymphoma: an updated analysis of Juliet, a global pivotal phase 2 trial of tisagenlecleucel. Blood 2018;132:1684. 
35. Locke FL, Ghobadi A, Jacobson CA, et al. Long-term safety and activity of axicabtagene ciloleucel in refractory large B-cell lymphoma (ZUMA-1): a single-arm, multicentre, phase 1-2 trial. Lancet Oncol 2019;20:31-42.

36. Locke FL, Neelapu SS, Bartlett NL, et al. Phase 1 results of ZUMA-1: a multicenter study of KTE-C19 anti-CD19 CAR $\mathrm{T}$ cell therapy in refractory aggressive lymphoma. Mol Ther 2017;25:285-95.

37. Crump M, Neelapu SS, Farooq U, et al. Outcomes in refractory diffuse large B-cell lymphoma: results from the international SCHOLAR-1 study. Blood 2017;130:1800-8.

38. Abramson JS, Gordon LI, Palomba ML, et al. Updated safety and long term clinical outcomes in TRANSCEND NHL 001, pivotal trial of lisocabtagene maraleucel (JCAR017) in R/R aggressive NHL. J Clin Oncol 2018;36:7505.

39. Xu J, Zhang Q, Tian K, et al. Current status and future prospects of the strategy of combining CAR-T with PD-1 blockade for antitumor therapy. Mol Med Rep 2018;17:2083-8.

40. Keir ME, Butte MJ, Freeman GJ, et al. PD-1 and its ligands in tolerance and immunity. Annu Rev Immunol 2008;26:677-704.

41. Baumeister SH, Freeman GJ, Dranoff G, et al. Coinhibitory pathways in immunotherapy for cancer. Annu Rev Immunol 2016;34:539-73.

42. Pardoll DM. The blockade of immune checkpoints in cancer immunotherapy. Nat Rev Cancer 2012;12:252-64.

43. Ribas A. Adaptive immune resistance: how cancer protects from immune attack. Cancer Discov 2015;5:915-9.

44. Simsek M, Tekin SB, Bilici M. Immunological agents used in cancer treatment. Eurasian J Med 2019;51:90.

45. Zolov SN, Rietberg SP, Bonifant CL. Programmed cell death protein 1 activation preferentially inhibits CD28. CAR-T cells. Cytotherapy 2018;20:1259-66.

46. Rupp LJ, Schumann K, Roybal KT, et al. CRISPR/Cas9mediated PD-1 disruption enhances anti-tumor efficacy of human chimeric antigen receptor T cells. Sci Rep 2017;7:737.

47. Chong EA, Melenhorst JJ, Lacey SF, et al. PD-1 blockade modulates chimeric antigen receptor (CAR)-modified T cells: refueling the CAR. Blood 2017;129:1039-41.

48. Cherkassky L, Morello A, Villena-Vargas J, et al. Human CAR T cells with cell-intrinsic PD-1 checkpoint blockade resist tumor-mediated inhibition. J Clin Invest 2016;126:3130-44.

49. Zhu H, Deng Q, Zhang R, et al. Effect of PD-1 inhibitor Nivolumab on the proliferation and cytotoxicity of anti-
CD19 chimeric antigen receptor T cells. Zhonghua Xue Ye Xue Za Zhi 2018;39:584.

50. Aalipour A, Advani RH. Bruton tyrosine kinase inhibitors: a promising novel targeted treatment for B cell lymphomas. Br J Haematol 2013;163:436-43.

51. Charalambous A, Schwarzbich MA, Witzens-Harig M. Ibrutinib. Recent Results Cancer Res 2018;212:133-68.

52. Deeks ED. Ibrutinib: a review in chronic lymphocytic leukaemia. Drugs 2017;77:225-36.

53. Ruella M, Kenderian SS, Shestova O, et al. The addition of the BTK inhibitor ibrutinib to anti-CD19 chimeric antigen receptor $\mathrm{T}$ cells (CART19) improves responses against mantle cell lymphoma. Clin Cancer Res 2016;22:2684-96.

54. Gill S, Frey NV, Hexner EO, et al. CD19 CAR-T cells combined with ibrutinib to induce complete remission in CLL. J Clin Oncol 2017;35:7509.

55. Klebanoff CA, Crompton JG, Leonardi AJ, et al. Inhibition of AKT signaling uncouples $\mathrm{T}$ cell differentiation from expansion for receptor-engineered adoptive immunotherapy. JCI Insight 2017;2:e95103.

56. Kochenderfer JN, Somerville RP, Lu T, et al. Lymphoma remissions caused by anti-CD19 chimeric antigen receptor $\mathrm{T}$ cells are associated with high serum interleukin-15 levels. J Clin Oncol 2017;35:1803-13.

57. Maus MV, Fraietta JA, Levine BL, et al. Adoptive immunotherapy for cancer or viruses. Annu Rev Immunol 2014;32:189-225.

58. Gattinoni L, Klebanoff CA, Restifo NP. Paths to stemness: building the ultimate antitumour T cell. Nat Rev Cancer 2012;12:671-84.

59. Zheng W, Jones LL, Geiger TL. Modulation of PI3K signaling to improve CAR T cell function. Oncotarget 2018;9:35807-8.

60. D'Souza WN, Chang CF, Fischer AM, et al. The Erk2 MAPK regulates CD8 T cell proliferation and survival. J Immunol 2008;181:7617-29.

61. Boni A, Cogdill AP, Dang P, et al. Selective BRAFV600E inhibition enhances $\mathrm{T}$-cell recognition of melanoma without affecting lymphocyte function. Cancer Res 2010;70:5213-9.

62. Macintyre AN, Finlay D, Preston G, et al. Protein kinase B controls transcriptional programs that direct cytotoxic $\mathrm{T}$ cell fate but is dispensable for $\mathrm{T}$ cell metabolism. Immunity 2011;34:224-36.

63. Sinclair LV, Finlay D, Feijoo C, et al. Phosphatidylinositol3-OH kinase and nutrient-sensing mTOR pathways control T lymphocyte trafficking. Nat Immunol 
2008;9:513-21.

64. Schubert ML, Hückelhoven A, Hoffmann JM, et al. Chimeric antigen receptor $\mathrm{T}$ cell therapy targeting CD19positive leukemia and lymphoma in the context of stem cell transplantation. Hum Gene Ther 2016;27:758-71.

65. Stock S, Übelhart R, Schubert ML, et al. Idelalisib for optimized CD19-specific chimeric antigen receptor $\mathrm{T}$ cells in chronic lymphocytic leukemia patients. Int J Cancer 2019;145:1312-24.

66. Klebanoff CA, Scott CD, Leonardi AJ, et al. Memory T cell-driven differentiation of naive cells impairs adoptive immunotherapy. J Clin Invest 2016;126:318-34.

67. Petersen CT, Hassan M, Morris AB, et al. Improving T-cell expansion and function for adoptive T-cell therapy using ex vivo treatment with PI3K $\delta$ inhibitors and VIP antagonists. Blood Adv 2018;2:210-23.

68. Bradner JE, West N, Grachan ML, et al. Chemical phylogenetics of histone deacetylases. Nat Chem Biol 2010;6:238-43.

69. Chu Y, Hochberg J, Yahr A, et al. Targeting CD20+ aggressive B-cell non-Hodgkin Lymphoma by anti-CD20 CAR mRNA-modified expanded natural killer cells in vitro and in NSG mice. Cancer Immunol Res 2015;3:333-44.

70. Xu Y, Li S, Wang Y, et al. Induced CD20 expression on B-cell malignant cells heightened the cytotoxic activity of chimeric antigen receptor engineered $\mathrm{T}$ cells. Hum Gene Ther 2019;30:497-510.

71. Chu Y, Yahr A, Huang B, et al. Romidepsin alone or in combination with anti-CD20 chimeric antigen receptor expanded natural killer cells targeting Burkitt lymphoma in vitro and in immunodeficient mice. Oncoimmunology 2017;6:e1341031.

72. Martelli M, Ferreri AJ, Agostinelli C, et al. Diffuse large B-cell lymphoma. Crit Rev Oncol Hematol 2013;87:146-71.

73. Roschewski M, Staudt LM, Wilson WH. Diffuse large B-cell lymphoma-treatment approaches in the molecular era. Nat Rev Clin Oncol 2014;11:12-23.

74. Camicia R, Winkler HC, Hassa PO. Novel drug targets for personalized precision medicine in relapsed/refractory diffuse large B-cell lymphoma: a comprehensive review. Mol Cancer 2015;14:207.

75. Jurczak W, Ochrem B, Giza A, et al. Role of rituximab in the first-line therapy of high-risk diffuse large B-cell lymphoma: a retrospective analysis by the Polish Lymphoma Research Group. Pol Arch Med Wewn 2015;125:741-8.

76. Rufener G. Preserved activity of CD20-specific chimeric antigen receptor-expressing $\mathrm{T}$ cells in the presence of rituximab. Cancer Immunol Res 2016;4:509-19.

77. James SE, Orgun NN, Tedder TF, et al. Antibodymediated B-cell depletion before adoptive immunotherapy with $\mathrm{T}$ cells expressing CD20-specific chimeric T-cell receptors facilitates eradication of leukemia in immunocompetent mice. Blood 2009;114:5454-63.

78. Mihara K, Yanagihara K, Takigahira M, et al. Synergistic and persistent effect of T-cell immunotherapy with antiCD19 or anti-CD38 chimeric receptor in conjunction with rituximab on B-cell non-Hodgkin lymphoma. Br J Haematol 2010;151:37-46.

79. Kumar SK, Dispenzieri A, Lacy MQ, et al. Continued improvement in survival in multiple myeloma: changes in early mortality and outcomes in older patients. Leukemia 2014;28:1122-8.

80. Franssen LE, Mutis T, Lokhorst HM, et al. Immunotherapy in myeloma: how far have we come? Ther Adv Hematol 2019;10:2040620718822660.

81. Chen N, Morello A, Tano Z, et al. CAR T-cell intrinsic PD-1 checkpoint blockade: A two-in-one approach for solid tumor immunotherapy. Oncoimmunology 2017;6:e1273302.

82. Sun S, Hao H, Yang G, et al. Immunotherapy with CARmodified T cells: Toxicities and overcoming strategies. J Immunol Res 2018;2018:2386187.
Cite this article as: Yang Y, Zhou J, Cao C, Cai P, Wang X, Chang C, Wang J, Zhang Q. Additional possibilities of chimeric antigen receptor T-cells in B-cell lymphoma: combination therapy. Transl Cancer Res 2020;9(11):7310-7322. doi: 10.21037/ tcr-20-72 